J. Clin. Chem. Clin. Biochem.

Vol. 19. 1981, pp. 89-97 \title{
Einfluß von Vorstufen von Faktor II oder Faktor X auf den Quick-Wert bei Verwendung von
Thromboplastinen verschiedener Hersteller
}

\author{
Von R. Averdunk und J. Grüner \\ Institut für Klinische Chemie und Klinische Biochemie der Freien Universität Berlin, Klinikum Steglitz
}

(Eingegangen am 26. März/25. August 1980)

Zusammenfassung: Die nicht-carboxylierte Vorstufe des Gerinnungsfaktors X beeinflußt die Bestimmung des QuickWertes bei Verwendung von Reagenzien verschiedener Hersteller unterschiedlich. Alle Reagenzien weisen eine gewisse Empfindlichkeit gegenüber nicht-carboxylierten Vorstufen auf.

Die Thromboplastine der Behringwerke und Thrombotest sind besonders empfindlich gegenüber nicht-carboxyliertem Gerinnungsfaktor X, während Simplastin von Goedecke und das Thromboplastin von Dade eine geringere und die Reagenzien von Boehringer und Hoffmann-La Roche eine etwas höhere Empfindlichkeit aufweisen. Nur die Unterschiede zwischen der ersten Gruppe (Behring Thromboplastine und Thrombotest) zu den anderen Thromboplastinen sind signifikant.

Der Quick-Wert, die Faktor X-Empfindlichkeit und die Empfindlichkeit gegenüber nicht-carboxyliertem Faktor X korrelieren bei Verwendung der getesteten Reagenzien gut.

Die Ergebnisse sind hinsichtlich der Richtigkeitskontrolle in Routine-Gerinnungslaboratorien und der Standardisierung der Throboplastinzeit wichtig.

\section{Sensitivity of several commercial thromboplastins to non-carboxylated clotting factors II and X}

Summary: All reagents for determination of thromboplastin time (Quick-test) tested so far are sensitive to non-carboxylated clotting factor $\mathrm{X}$, and sensitivity varies, depending on the reagent. The thromboplastins of Behring and Thrombotest are very sensitive. The preparations of La-Roche and Boehringer are somewhat more sensitive than those of Dade und Goedecke. Only the differences between the first group (Behring, Thrombotest) and the others are significant.

There is a good correlation between factor $\mathbf{X}$ sensitivity, prothrombin time and non-carboxylated clotting factor $\mathbf{X}$ sensitivity, with all reagents. These results may be of significance for quality control and standardization of the prothrombin time.

\section{Einführung}

Vitamin K-Antägonisten verursachen eine Abnahme der Konzentration vollständig carboxylierter und eine $\mathrm{Zu}$ nahme der Konzentration von nicht carboxylierten Gerinnungsfaktoren, die als PIVKA (Protein Induced by Vitamin K Absence) bezeichnet wurden $(1,2)$. Das unter VitaminK-Mangel entstehende, nicht carboxylierte Prothrombin läßt sich nicht an Bariumcitrat adsorbieren und besitzt nur eine sehr geringe Calcium- und Phospholipid-Bindungsfähigkeit. Ës hat jedoch die gleichen Antigenmerkmale wie normales Prothrombin (2-6).

Schon bevor das Auftreten eines nicht carboxylierten Prothrombins im Plasma bei Vitamin K-Mangel bekannt war, hatte Hemker durch kinetische Untersuchungen das Vorliegen eines Stoffes gefordert, der eine Hemmwirkung bei der Bestimmung der Thromboplastinzeit ausübt. Hemker benutzte für die Dạstellung dieser Hemmung ein modifiziertes Lineweaver-Burk-Diagramm. Es wurde gefunden, daß „PIVKA“ bei der Bestimmung der Prothrombinzeit als kompetitiver Hemmstoff wirkt (1).

Die Empfindlichkeit verschiedener Thromboplastine auf nicht carboxylierte Gerinnungsfaktoren ist möglicherweise bei der Antikoagulation mit Vitamin K-Antagonisten wichtig, da sie gerinnungshemmend wirken könnten. Nur ein Reagenz mit hoher Empfindlichkeit gegenüber nicht-carboxylierten Gerinnungsfaktoren wäre dann geeignet, ein Blutungsrisiko anzuzeigen.

Bẹi der notwendigen Standardisierung des Quick-Wertes ist es erforderlich, auch hinsichtlich der Empfindlichkeit gegenüber nicht-carboxylierten Gerinnungsfaktoren zu standardisieren. Bei der Richtigkeitskontrolle des QuickWertes müssen nicht-carboxylierte Gerinnungsfaktoren ebenfalls berücksichtigt werden. 
In der vorliegenden Arbeit wurden aus Gründen der Standardisierung, Qualitätskontrolle und Definition der Aussagekraft sieben Thromboplastinreagenzien verschiedener Hersteller auf ihre Gesamt-Empfindlichkeit gegenüber nicht-carboxylierten Gerinnungsfaktoren und ihre Empfindlichkeit gegenüber nicht-carboxylierten $\mathrm{Ge}$ rinnungsfaktoren II und $\mathrm{X}$ getestet.

\section{Material und Methoden}

Als Ausgangsmaterial diente Citratplasma. Nach Abtrennung der Erythrocyten $(1500 \mathrm{~g} ; 10 \mathrm{~min})$ wurde für $25 \mathrm{~min}$ bei $15000 \mathrm{~g}$ in einer gekühlten Sorvall RC3, Rotor SS 34, zentrifugiert, um thrombocytenfreies Plasma zu erhalten.

Das Eintreten der Gerinnung wurde mit einem Koagulometer der Firma Schnittger, Göttingen, bei $37^{\circ} \mathrm{C}$ gemessen.

Zur Erstellung der Normalkurven wurde gepooltes Plasma von mindestens 8 gesunden Personen verwendet. Nicht-carboxylierte Gerinnungsfaktoren enthaltendes Plasma wurde von Patienten gewonnen, die seit mindestens 6 Monaten mit Phenprocumon (Marcumar ${ }^{\circledR}$ ) behandelt wurden.

\section{Thromboplastinzeitbestimmung}

Es wurde für jedes Thromboplastin eine Standardkurve erstellt, um die Umrechnung von Sekunden auf Normprozent zu ermöglichen. Die Standardkurve wurde durch Verdünnung eines Gemisches von Plasmen 8 gesunder Probanden mit $0,15 \mathrm{~mol} / 1 \mathrm{NaCl}$ an drei verschiedenen Tagen aufgestellt. Es wurden so Meßzahlen mit $100,80,50,20,14,12,5$ und $10 \%$ festgelegt. Folgende Thromboplastine wurden verwendet:

1. Calciumthromboplastin La ROCHE, Firma Hoffmann-La Roche, Basel;

2. Calciumthromboplastin Dade, Firma Merz und Dade, München;

3. Calciumthromboplastin Behring, Firma Behringwerke, Marburg/Lahn;

4. Thromborel, Firma Behringwerke, Marburg/Lahn;

5. Simplastin, Firma Goedecke, Freiburg/Breisgau;

6. Calciumthromboplastin Boehringer, Firma Boehringer, Mannheim;

7. Thrombotest, Firma Nyegaard, Oslo.

Nach Vorgabe von $0,1 \mathrm{ml}$ Plasma bzw. Plasmaverdünnung und 30 Sekunden Vorinkubationszeit wurde die Reaktion durch $\mathrm{Zu}$ gabe von $0,2 \mathrm{ml}$ eines der Thromboplastine gestartet. Für den Testansatz mit Thrombotest wurde das Plasma 3: 5 mit bidest. Wasser verdünnt, nach Vorgabe von $0,25 \mathrm{ml}$ Plasmaverdünnung $0,05 \mathrm{ml}$ Thrombotestreagenz zugegeben.

\section{Bestimmung der Aktivität nicht-carboxylierter Gerinnungsfak-
toren}

Zur Ermittlung der Aktivität nicht-carboxylierter Gerinnungsfaktoren wurde das von Hemker (1) modifizierte LineweaverBurk-Diagramm herangezogen:

$\mathrm{t}_{\mathrm{c}} \times \mathrm{h}=\frac{1}{[\mathrm{E}]}+\frac{1}{[\mathrm{~S}]}+\frac{K_{\mathrm{m}}}{[\mathrm{ES}]}$

$t_{c}=$ Gerinnungszeit

$\mathrm{h} \quad=$ Konstante

[E] = Enzymkonzentration

[S] = Substratkonzentration zu Reaktionsbeginn

$K_{\mathrm{m}} \quad=$ Michaelis-Konstante der Reaktion von E mit S

Zur Bestimmung der Hemmung durch nicht-carboxylierten $\mathrm{Ge}$ rinnungsfaktor $\mathbf{X}$ wurden Verdünnungen des Citratplasmas mit Faktor X-Mangelplasma bei D gleich $1 ; 1,25 ; 2 ; 5 ; 7 ; 8$ und 10 erstellt. „D“ bezeichnet dabei den Reziprokwert des Quotienten von ursprünglichem Plasmavolumen zu Endvolumen nach Verdünnung vor Reagenzzugabe; es entspricht dem Kehrwert der Plasmaverdünnung: Verdünnung $1: 10$ ergibt $D=10$.

Nachdem zunächst die Thromboplastinzeiten gepoolter Normalplasmen dieser Verdünnungen ermittelt wurden, erfolgte die Mes- sung der Verdünnungen von Plasmen Phenprocumon-behandelter Probanden in Doppelbestimmungen.

Die Thromboplastinzeiten wurden in ein $t_{c}$-D-Diagramm eingetragen und die Regressionsgerade für jedes Plasma wurde ermittelt $(1,2)$.

Die relative Menge des kompetitiven Hemmstoffes (= nicht-carboxylierten Gerinnungsfaktors) wurde bestimmt, indem eine Parallele zur Abszisse durch den Schnittpunkt der Geraden aus Normalplasma mit der Ordinate gezogen wurde. Der gesuchte Abschnitt I auf dieser Parallelen zur Absżisse wird durch die Schnittpunkte mit der Ordinate und mit der Geraden aus nichtcarboxylierten Gerinnungsfaktoren enthaltenden Plasmaverdïnnungen begrenzt (Abb. 1). Die Maßeinheit entspricht der Strekke $\mathrm{D}=1$ und damit einer Strecke von $1 \mathrm{~cm}$. Die Hemmung I wird durch Einsetzen des $t_{c}$-Wertes der Normalgeraden bei $D=0$ in die Gleichung der Geraden des gemessenen nicht-carboxylierte Gerinnungsfaktoren enthaltenden Plasmas errechnet.

Die Aktivität von nicht-carboxyliertem Gerinnungsfaktor II wurde unter Verwendung eines Faktor-II-Mangelplasmas in einem für nichtcarboxylierten Gerinnungsfaktor $\mathrm{X}$ beschriebenen, analogen Vorgehen bestimmt.

Bei der Bestimmung der Gesamt-Aktivität nicht-carboxylierter Gerinnungsfaktoren würde mit $O$ wrens-Puffer verdünnt.

\section{Ergebnisse}

\section{Bestimmung der Aktivität nicht-carboxylierter Gerin- nungsfaktoren mit Puffer als Verdünnungsreagenz}

Verdünnt man die Plasmen von gesunden Probanden oder von Patienten unter Phenprocumon (Marcumar ${ }^{\circledR}$ ).

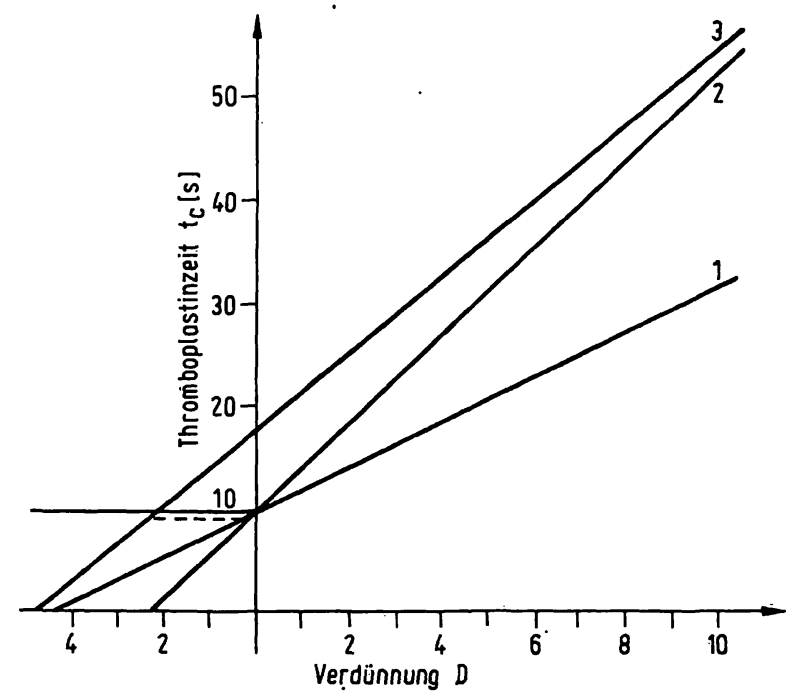

Abb. 1. Beispiel für die graphische Ermittlung der Hemmung I durch Plasma, das aufgrund einer Therapie mit Phenprocumon nichtcarboxylierte Gerinnungsfaktoren enthält: $t_{c}$-D-Diagramm

1: Gerade für Normalplasma

2: Gerade für das Plasma eines Patienten mit Lebercirrhose zum Vergleich

3: Gerade für das Plasma eines Patienten unter Phenprocumon (Marcumar ${ }^{\circledR}$ )-Therapie

Verdünnung des Plasmas jeweils mit Faktor X-MangelPlasma.

Auswertung

Vom Schnittpunkt der Geraden für Normalplasmà mit der Ordinate wird eine Parallele zur Abszisse eingezeichnet. Die Distanz der Schnittpunkte der Geraden für Normalplasmana und der für das zu untersuchende Plasma wird ermittelt (gestrichelt). Hier ergibt sich éin Hemmwert $I=2,2$. 
Behandlung mit Owrens-Puffer, mißt die Thromboplastinzeiten und trägt diese gegen die Verdünnung D auf, so erhält man eine Schar von Geraden mit unterschiedlichen Steigungen und Ordinatenabschnitten (Abb. 2a bis 2c). Die unterschiedlichen Steigungen sind durch die Verringerung der Gerinnungsfaktoren-Konzentrationen bedingt, die in den Plasmen in unterschiedlicher Ausgangskonzentration vorliegen. Die Steigungen der Geraden variieren mit den einzelnen Reagenzien. Thrombotest zeigt bei allen Plasmen die stärkste Steigung, reagiert also besonders empfindlich auf eine Abnahme der Faktorenkonzentrationen.

Die Schnittpunkte der Geraden aus Normalplasmen liegen nur beim Behring- und Thromotest-Reagenz in einem beträchtlichen Abstand von den Schnittpunkten der Geraden aus Plasmaverdünnungen von mit Phenprocumon (Marcumar $^{\circledR}$ ) behandelten Patienten (Abb. 2a + 2b). Bei diesen beiden Reagenzien sind auch die Ordinatenabschnitte über eine relativ weite Strecke verstreut. Bei Verwendung anderer Thromboplastine schneiden die Geraden aus Normalplasmaverdünnungen und der Verdünnung aus den Phenprocumonplasmen die Ordinate in einem sehr engen Bereich.

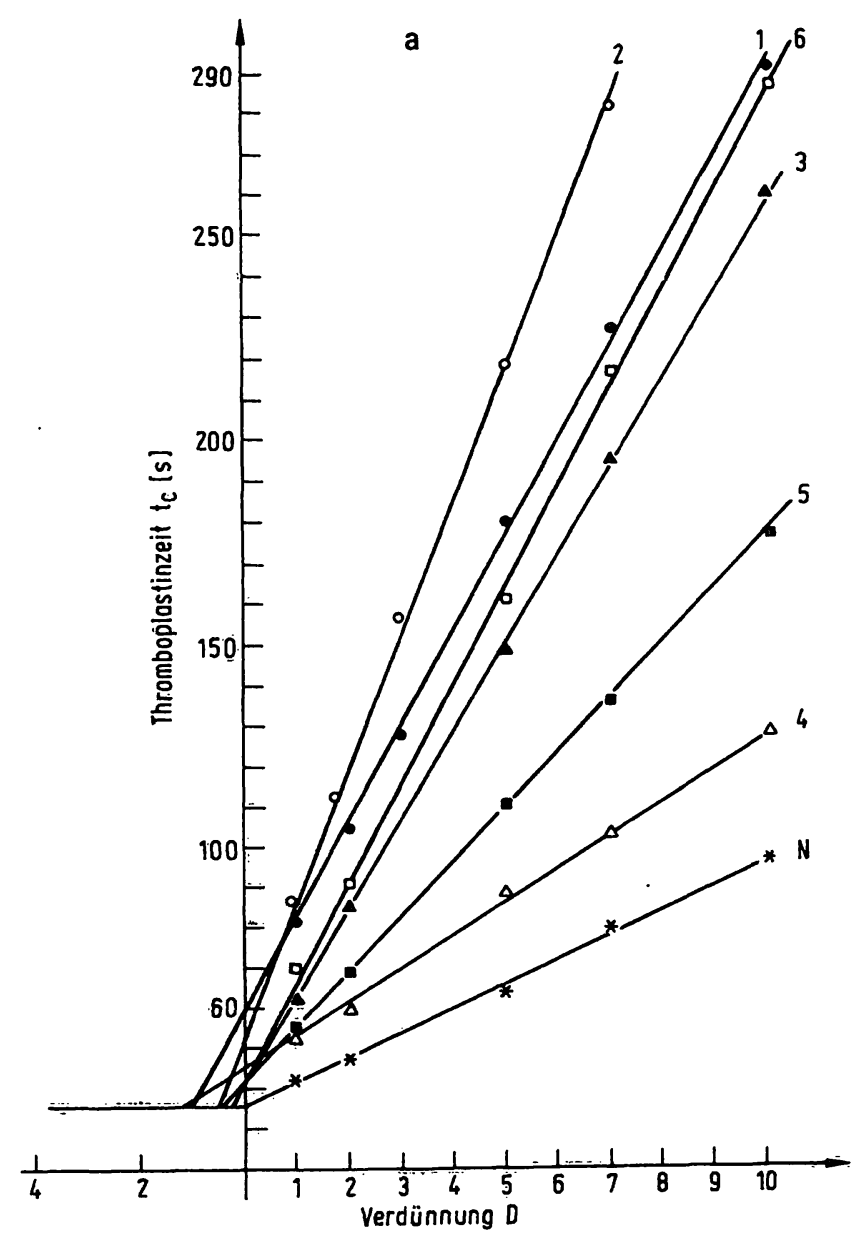

Abb. 2a-2c. $t_{c}$-D-Diagramme nach Verdünnung der Plasmen mit Puffer.

In den Diagrammen wurde die Thromboplastinzeit $t_{c}$ gegen die Verdunnung $D$ aufgetragen. Es wurden die Plasmen von Patienten unter Phenprocumon-
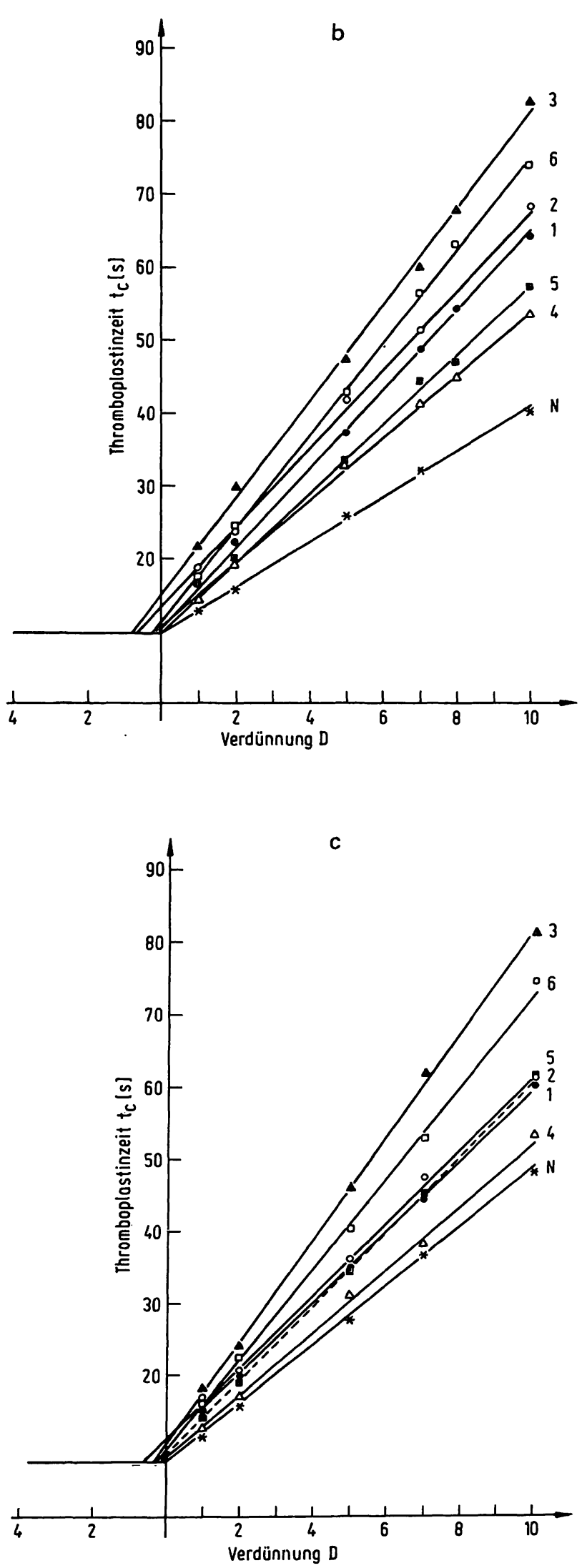

therapie mit Owrens-Puffer verdünnt.

Als Testreagenz wurde

a) Thrombotest,

b) Calciumthromboplast in der Behringwerke,

c) Thromboplastin von Dade verwendet.

$\mathrm{N}=$ Normalplasma.

1-6 Plasmen Phenprocumon-behandelter Patienten. 
Bei den einzelnen Thromboplastinen erhält man deutlich unterschiedliche Werte (Tab. 1) für I (Maß für die durch nicht-carboxylierte Faktoren bedingte Hemmung). Dabei ist die Hemmwirkung bei allen Reagenzien relativ gering. Die Durchschnittswerte von I verschiedener Thromboplastine, gewonnen aus mehreren Phenprocumonplasmen, lassen eine Reihenfolge der Gesamtempfindlichkeit gegenüber nicht-carboxylierten Gerinnungsfaktoren einzelner Reagenzien erkennen. Danach findet sich eine besonders hohe Hemmwirkung durch die nicht-carboxylierten Gerinnungsfaktoren bei den Thromboplastinen der Behringwerke und Thrombotest, eine etwas weniger ausgeprägte bei Simplastin und Dade und eine eventuell noch geringere Hemmung der Thromboplastine von Roche und Boehringer.

Gegen die Verwendung von Puffer bei der Verdünnung der Phenprocumonplasmen läßt sich einwenden, daß sämtliche Faktoren gleichmäßig verdünnt werden und so die Reaktionsgeschwindigkeit von der verminderten Konzentration aller Gerinnungsfaktoren bestimmt wird, besonders des Fibrinogen. Da eine Aktivität nicht-carboxylierter Gerinnungsfaktoren gegen die Faktoren II, VII, IX und $\mathrm{X}$ beschrieben wurde, würde die Hemmung eines dieser Gerinnungsfaktoren schon eine Verlängerung der I-Strecke bewirken.

Bei Verwendung von Puffer zur Verdünnung ist es also nicht möglich, zwischen nicht-carboxylierten Gerinnungsfaktoren II, X und evtl. VII zu unterscheiden, sondern es würde sich bestenfalls eine Gesamtübersicht über die Aktivität nicht-carboxylierter Gerinnungsfaktoren ergeben. Gegen eine Verdünnung mit Puffer spricht auch die kürzli sh gemachte Beobachtung, daß es zu einem sehr schnellen Abfall der Konzentration von nicht-carboxyliertem Gerinnungsfaktor VII bei hohen, mit Puffer angesetzten Plasmaverdünnungen kommen kann (18).

In den nächsten Versuchsserien wurden deshalb Mangelplasmen zur Verdünnung der Phenprocumonplasmen verwendet. Dadurch soll erreicht werden, daß die Konzentration aller Faktoren bis auf den zu untersuchenden konstant bleibt. Verdünnt man nun die Phenprocumonplasmen mit Faktor II-Mangelplasma und mißt die Throm- boplastinzeit, so findet sich, wie aus den Abbildungen $3 a-3 c$ hervorgeht, keine lineare Funktion zwischen der Thromboplastinzeit $t_{c}$ und der Verdünnung $D$. Bei $\mathrm{D}=1$ und 2 ist eine Verkürzung der Thromboplastinzeit, d. h. eine Erhöhung der Reaktionsgeschwindigkeit, im Vergleich zur unverdünnten Plasmaprobe zu erkennen. Erst bei einer Verdünnung von $D>2$ wird $t_{c}$ wieder grösser als $t_{c}$ der unverdünnten Probe $(D=1)$, um im $t_{c}-D$ Diagramm dann annähernd linear zu verlaufen.

Das Verhalten der Thromboplastinżeit der Phenprocumonplasmen nach Verdünnung mit Faktor II-Mangelplasma läßt sich dadurch erklären, daß dieses aktivierbaren Faktor X enthält. Ess wird also dem Phenprocumonplasma, das wenig aktivierbaren Faktor X enthält, mit dem Reagenzienplasma Faktor X zugegeben. Dạ Faktor $\mathrm{X}$ der Gerinnungsfaktor ist, der die Geschwindigkeit der Gesamtreaktion bestimmt (9), tritt eine Verkürzung von $t_{c}$, also eine Erhöhung der Reaktionsgeschwindigkeit, ein. Faktor II wird in der hier benutzten Versuchsanordnung erst bei starker Verdünnung zum geschwindigkeitsbestimmenden Faktor der Gesamtreaktion, was sich in den Abbildungen $2 a-2 c$ in einem linearen Kurvenanteil auch zwischen $D=7$ und $D=10$ ausdrückt. Da diese Geraden jedoch nicht die Werte für unverdünntes Normalplasma $(D \doteq 1)$ tangieren, kann diese Versuchsanordnung auch nicht zur Bestimmung von nicht-carboxyliertem Faktor II herangezogen werden.

Verdünnt man nun die Plasmen mit Faktor X-Mangelplasmen, so erhält man, ähnlich wie bei der Verdünnung mit Puffer, wieder Geraden mit unterschiedlichen Steigun= gen und Ordinatenabschnitten (Abb. 4a-4c).

In Tabelle 2 sind die Werte für die Hemmung I angegeben. Mit den beiden Behringwerk-Thromboplastinen und dem Thrombotestreagenz wird bei allen Plasmen eine starke Hemmung durch die nicht vollständig carboxylierte Faktor X-Vorstufe registriert. Die Empfindlichkeit gegenüber nicht-carboxyliertem Faktor $\mathrm{X}$ innerhalb dieser Gruppe ist nicht signifikant unterschiedlich (Tab. 3).

Eine geringere Hemmwirkung durch nicht-carboxylierte Gerinnungsfaktoren wird mit den Thromboplastinen von

Tab. 1. Durch nicht-carboxylierte Gerinnungsfaktoren bedingte Hemmung in verschiedenen Plasmen nach Verdünnung mit Owrens-Puffer.

In 6 Plasmen Phenprocumon-behandelter Patienten wurden die Thromboplastinzeiten mit Reagenżien verschiedener Hersteller nach Verdünnung der Plasmen mit Owrens-Puffer $(1: 10,1: 5,1: 2,1: 1,25)$ und unverdünnt bestimmt.

In der Tabelle sind die Werte für $I$ angegeben.

\begin{tabular}{|c|c|c|c|c|c|c|c|}
\hline \multirow[b]{2}{*}{$\begin{array}{l}\text { Plasma } \\
\text { Nr. } \\
\end{array}$} & \multicolumn{7}{|c|}{ Thromboplastin } \\
\hline & Roche & Dade & Behring & Thromborel & Simplastin & Boehringer & Thrombotest \\
\hline $\begin{array}{l}1 \\
2 \\
3 \\
4 \\
5 \\
6\end{array}$ & $\begin{array}{l}- \\
\overline{0} \\
0,37 \\
0,09 \\
0,31 \\
0,15\end{array}$ & $\begin{array}{l}0,60 \\
0,44 \\
0,29 \\
0,29 \\
0,20 \\
0,20\end{array}$ & $\begin{array}{l}1,47 \\
0,56 \\
0,77 \\
0,26 \\
0,10 \\
0,22\end{array}$ & $\begin{array}{l}- \\
\overline{0} \\
0,10 \\
0,21 \\
0,31 \\
1,13\end{array}$ & $\begin{array}{l}\cdot \overline{-} \\
\overline{0,55} \\
0,75 \\
0,27 \\
0,13\end{array}$ & $\begin{array}{l}0,23 \\
0,20 \\
0,20 \\
0,29 \\
0,13 \\
0,10\end{array}$ & $\begin{array}{l}0,49 \\
0,50 \\
0,31 \\
1,23 \\
0,40 \\
0,18\end{array}$ \\
\hline $\bar{x}$ & 0,24 & 0,34 & 0,56 & 0,44 & 0,42 & 0,19 & 0,52 \\
\hline
\end{tabular}



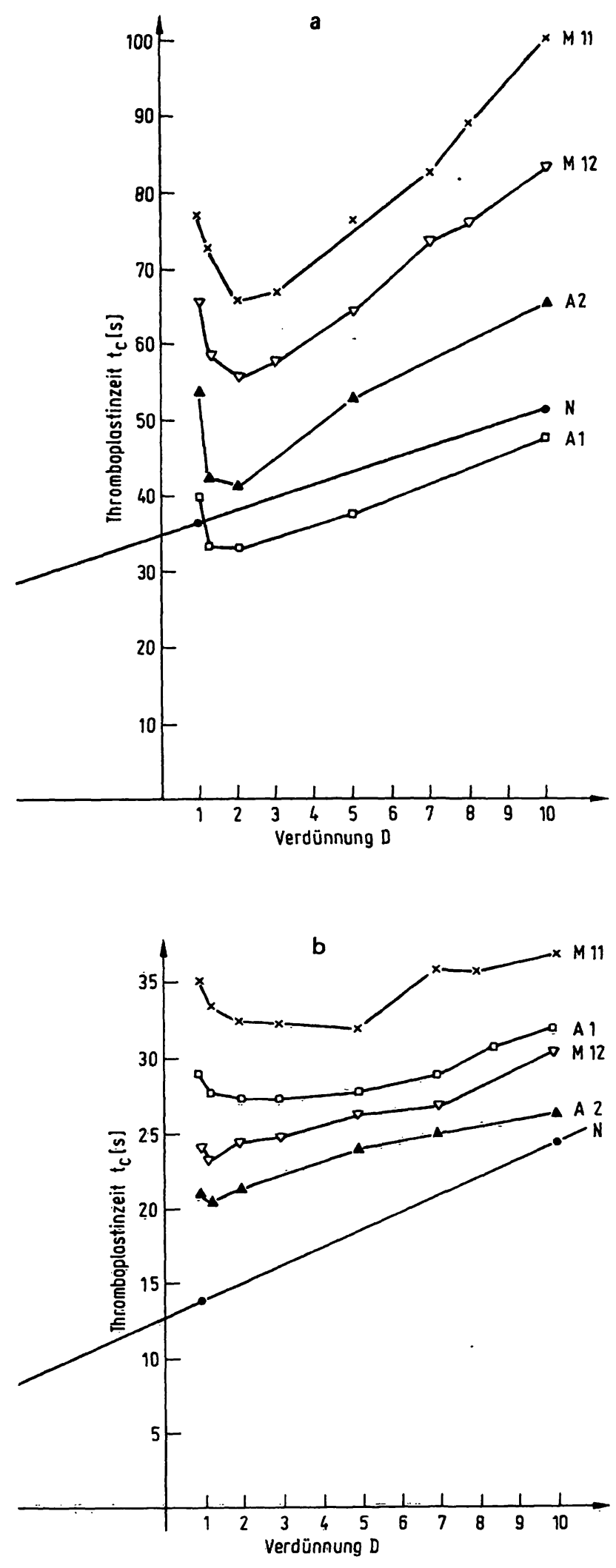

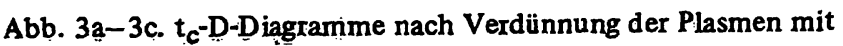
Faktor İl-Mangelplasmen.

Die Plạsmen vợ Patienten unter Phenprocumontherapie wurden mit Faktor II-Mangelplasma der Firma Dade verdünnt.

Als Testreagenz wurde

a) Thrombotest,

b) Calciumthromboplastin der Behringwerke,

c) Thromboplastin von Dade verwendet.
Boehringer und Roche registriert. Die Unterschiede gegenüber der ersten Gruppe sind signifikant (Tab. 3).

Noch weniger ausgeprägt ist die Hemmung durch nichtcarboxylierten Faktor X bei den Reagenzien Simplastin und Dade. Hier ergibt sich ein hoch signifikanter Unterschied zur ersten Gruppe, während die Unterschiedlichkeit zwischen der zweiten und dritten Gruppe bei Dade/ Roche und Simplastin/Roche nicht signifikant ist.

In Tabelle 2a wird die Reihenfolge der Empfindlichkeit gegenüber nicht-carboxylierten Gerinnungsfaktoren für die einzelnen Plasmen wiedergegeben. Es wäre zu erwarten, daß ein Plasma mit dem höchsten Gehalt an nichtcarboxyliertem Faktor $\mathrm{X}$ von allen Thromboplastinen, wenn auch mit unterschiedlicher Empfindlichkeit, als Plasma mit dem größten Wert für I reagiert. Die Reihenfolge variiert aber bei den einzelnen Plasmen. So wird der höhste I-Wert bei Plasma 1 (Tab. 2a) durch das Behringwerk-Thromboplastin, Plasma 3 durch Thrombotest, Plasma 4 durch Thromborel gefunden. Es finden sich aber immer die höchsten Werte für I in den drei als besonders empfindlich angegebenen Reagenzien. Einzige Ausnahme ist Plasma 2 und 5, bei denen der vierthöchste IWert, einmal mit Thrombotest und einmal mit dem Behringwerk-Calcium-Thromboplastin, bestimmt wurde.

Die Tatsache, daß nicht bei allen Plasmen die gleiche Reihenfolge des I-Wertes zu finden ist, läßt sich nur durch einen methodisch bedingten hohen, zufälligen Fehler erklären. Dieser Fehler führt auch dazu, daß nur die Unterschiede zwischen den sehr empfindlichen Thromboplastinen (Behringwerke, Thrombotest) zu den anderen Thromboplastinen signifikant sind.

Wenn nun, wie die Tabelle 2 zeigt, die verschiedenen Thromboplastine unterschiedlich stark auf die Hemmwirkung von nicht-carboxyliertem Faktor $\mathrm{X}$ reagieren, so müßten auch bei der quantitativen Bestimmung des Fak-

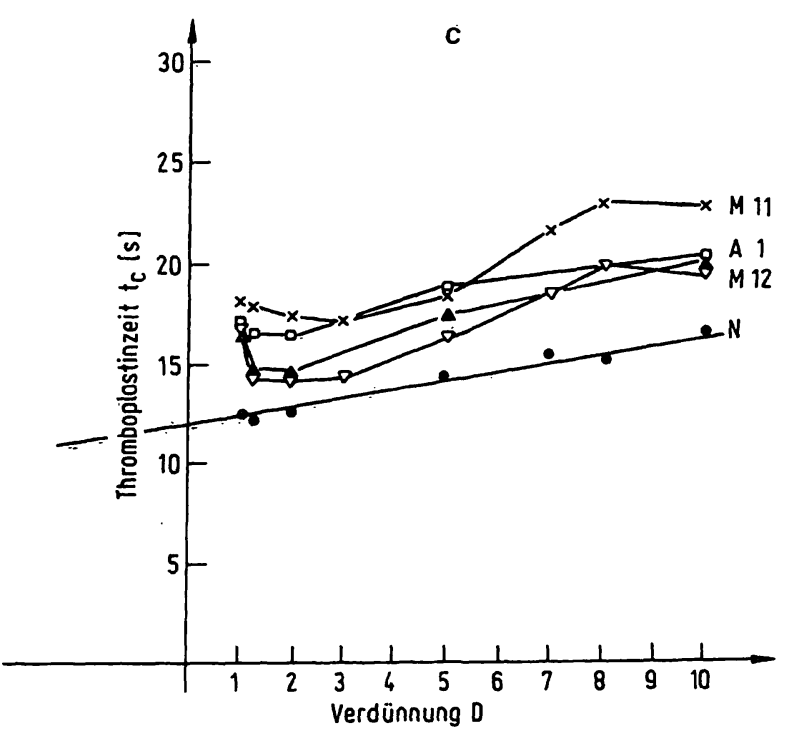

$\mathrm{N}=$ Normalplasma.

$A_{1}, A_{2}, M_{11}, M_{12}$ Plasmen Phenprocumon-behandelter Patienten. 

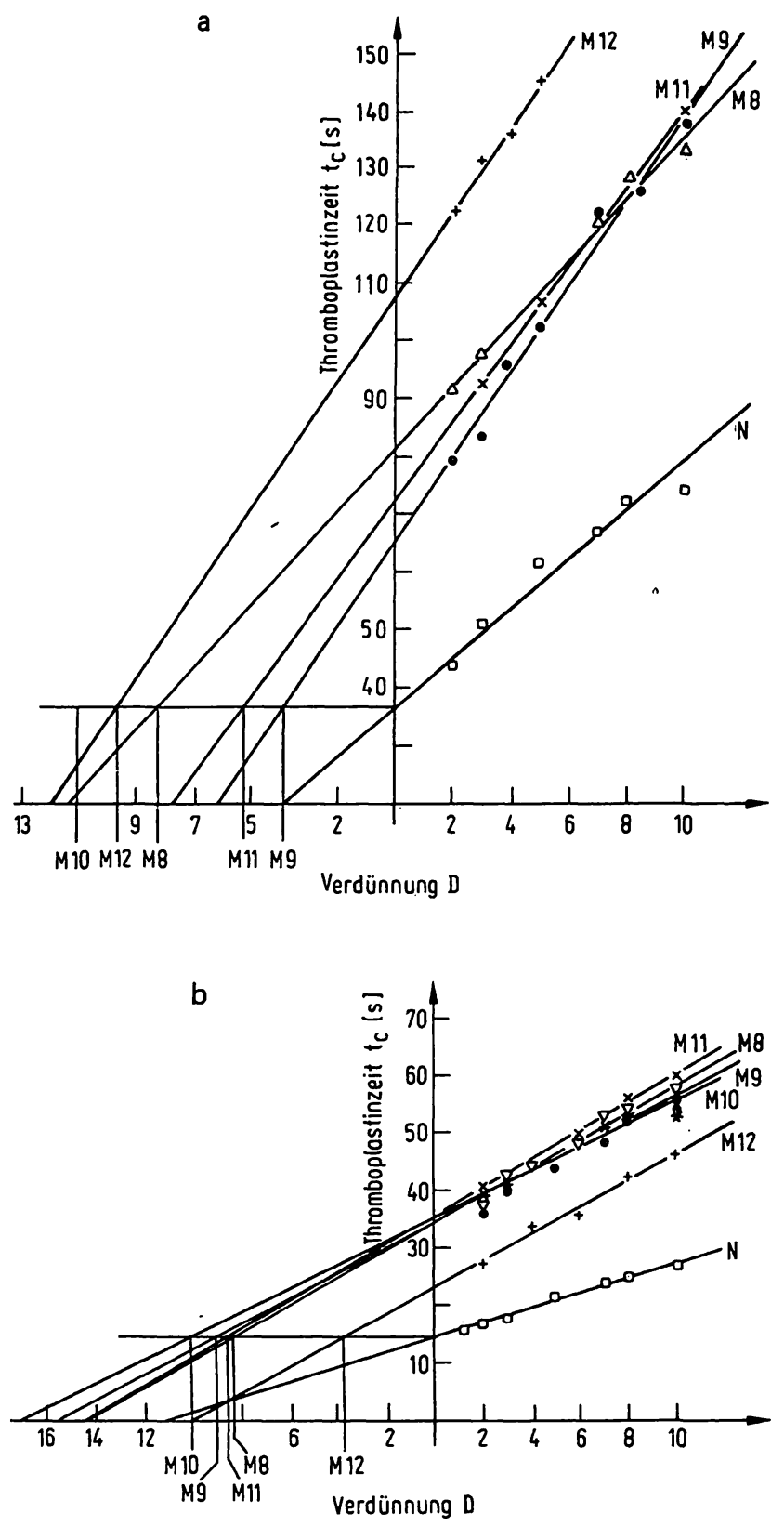

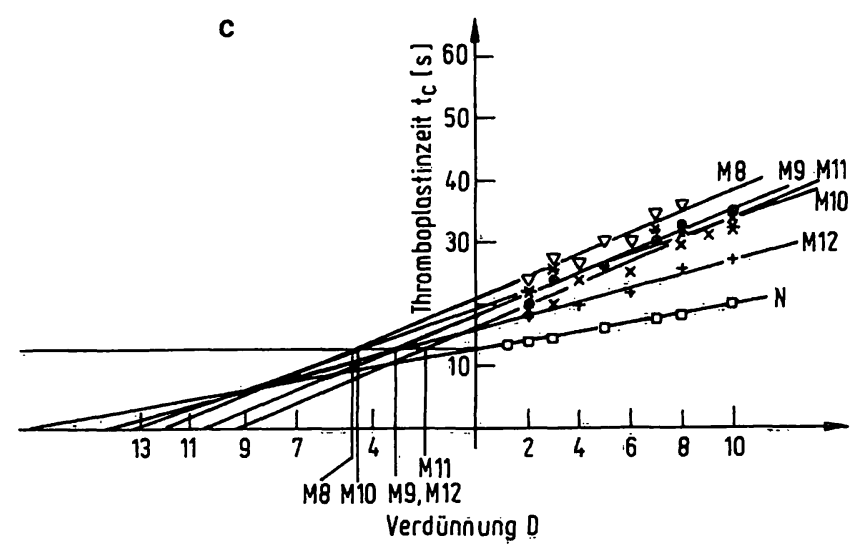

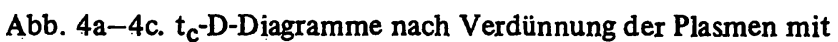
Faktor X-Mangelplasmà.

Die Plasmen der Patienten unter Phenprocumontherapie wurden mit Faktor X-Mangelplasma der Firma Dade verdünnt.

Als Testreagenz wurde

a) Thrombotest,

b) Calciumthromboplastin der Behringwerke,

c) Thromboplastin von Dade verwendet.

$\mathrm{N}=$ Normalplasma.

$\mathrm{M}_{8}-\mathrm{M}_{12}$ Plasmen Phenprocumon-behandelter Patienten.

Tab. 2a. Auflistung der I-Werte

Die Werte aus Tabelle 2 wurden nach ihrer absoluten Höhe aufgelistet. Das Thromboplastin, das den höchsten I-Wert in einem Plasma anzeigt, erhält den Wert 1, das Thromboplastin, das den zweithöchsten Wert anzeigt, die Zahl 2, usw.

\begin{tabular}{lcccccccccc}
\hline & \multicolumn{1}{c}{ Plasma-Nr. } \\
Reagenz & X & 1 & 2 & 3 & 4 & 5 & 6 & 7 & 8 & 11 \\
\hline Behringwerke & 1 & 1 & 1 & 2 & 2 & 4 & 1 & 2 & 2 & 1 \\
Thromborel & 2 & 2 & 2 & 3 & 1 & 2 & 2 & 1 & 3 & 2 \\
Thrombotest & 3 & 3 & 4 & 1 & 3 & 1 & 3 & 3 & 1 & 3 \\
Boehringer & 4 & 4 & 3 & 5 & 5 & 3 & 4 & 6 & 5 & 5 \\
Roche & 5 & 7 & 5 & 6 & 4 & 5 & 7 & 4 & 6 & 4 \\
Simplastin & 6 & 6 & 7 & 7 & 6 & 7 & 6 & 7 & 4 & 6 \\
Dade & 7 & 5 & 6 & 4 & 7 & 6 & 5 & 5 & 7 & 7 \\
\hline
\end{tabular}

Tab. 2. Bestimmung der Aktivität nicht-carboxylierter Gerinnungsfaktoren in Plasmen Phenprocumon-behandelter Patienten nach Verdünnung mit Faktor X-Mangelplasma.

In den Plasmen von 12 verschiedenen, unter Phenprocumontherapie stehenden Patienten wurden nach Verdünnung dieser Plasmen im Verhältnis $1: 10,1: 5,1: 2,1: 1,25$ und einer unverdünnten Probe mit Hilfe verschiedener Thromboplastine die Thromboplastinzeiten bestimmt.

In der Tabelle sind die Werte für die Hemmwirkung I angegeben.

\begin{tabular}{|c|c|c|c|c|c|c|c|}
\hline \multirow[b]{2}{*}{$\begin{array}{l}\text { Plasma } \\
\text { Nr. } \\
\end{array}$} & \multicolumn{7}{|c|}{ Thromboplastin } \\
\hline & Roche & Dade & Behring & Thromborel & Simplastin & Boehringer & Thrombotest \\
\hline $\begin{array}{r}1 \\
2 \\
3 \\
4 \\
5 \\
6 \\
7 \\
8 \\
9 \\
10 \\
11 \\
12\end{array}$ & $\begin{array}{l}3,05 \\
3,89 \\
3,59 \\
4,71 \\
2,51 \\
1,44 \\
2,86 \\
2,62 \\
- \\
- \\
3,03 \\
2,06\end{array}$ & $\begin{array}{l}4,09 \\
3,13 \\
5,12 \\
1,88 \\
1,94 \\
1,52 \\
2,39 \\
2,09 \\
1,45 \\
1,47 \\
1,64 \\
1,21\end{array}$ & $\begin{array}{l}7,78 \\
8,28 \\
8,25 \\
7,16 \\
3,86 \\
3,04 \\
3,80 \\
4,03 \\
4,72 \\
5,53 \\
4,22 \\
1,06\end{array}$ & $\begin{array}{l}7,16 \\
5,76 \\
6,73 \\
7,92 \\
5,15 \\
3,00 \\
4,32 \\
4,02 \\
- \\
- \\
4,07 \\
1,03\end{array}$ & $\begin{array}{l}3,58 \\
3,30 \\
3,50 \\
3,08 \\
1,73 \\
1,14 \\
1,27 \\
2,90 \\
- \\
- \\
1,74 \\
1,73\end{array}$ & $\begin{array}{l}4,57 \\
5,87 \\
6,08 \\
4,69 \\
4,09 \\
1,84 \\
2,24 \\
2,76 \\
2,15 \\
1,79 \\
2,58 \\
1,35\end{array}$ & $\begin{array}{l}7,56 \\
3,86 \\
8,97 \\
5,52 \\
8,90 \\
2,10 \\
3,30 \\
5,39 \\
2,67 \\
2,23 \\
3,77 \\
0,81\end{array}$ \\
\hline$\overline{\mathrm{x}}$ & 2,98 & 2,33 & 5,14 & 4,92 & 2,40 & 3,25 & $4,59^{\circ}$ \\
\hline
\end{tabular}


Tab. 3. Signifikanzberechnung der Wirkung von nicht-carboxyliertem Faktor $\mathbf{X}$.

Die Signifikanzberechnung nach Student vergleicht die durch nicht-carboxylierten Faktor X bedingten Hemmungen (Tab. 2), gemessen mit verschiedenen Thromboplastinzeiten.

\begin{tabular}{lllllll}
\hline & Dade & Behring & Thromborel & Simplastin & Boehringer & Thrombotest \\
Roche & 0,25 & 0,005 & 0,004 & 0,05 & 0,15 & 0,04 \\
& Dade & 0,001 & 0,001 & 0,7 & 0,01 & 0,03 \\
& Behring & 0,6 & 0,001 & 0,001 & 0,4 \\
& & Thromborel & 0,001 & 0,003 & 0,85 \\
& & & Simplastin & 0,005 & 0,005 \\
& & & & Boehringer & 0,025 \\
\hline
\end{tabular}

Tab. 4. Faktor X-Aktivitäten und Quick-Werte verschiedener Plasmen von unter Phenprocumontherapie stehenden Patienten, gemessen mit Thro mboplastinreagenzien verschiedener Hersteller.

Die Bestimmung des Quick-Wertes erfolgte nach Angabe der Hersteller. Zur Bestimmung der Faktor X-Konzentration der einzelnen Plasmen wurde Faktor X-Mangelplasma der Firma Dade als Reagenzienplasma verwendet. Den Werten der Tabelle liegen Dreifachbestimmungen zugrunde.

Angabe in Prozent der Norm. RVV = Russel's Viper Venom.

\begin{tabular}{|c|c|c|c|c|c|c|c|c|c|c|c|c|c|c|c|}
\hline \multirow[b]{2}{*}{$\begin{array}{l}\text { Plas- } \\
\text { ma } \\
\text { Nr. }\end{array}$} & \multicolumn{15}{|c|}{ Thromboplastin } \\
\hline & $\begin{array}{l}\text { Roche } \\
\text { Quick- } \\
\text { Wert }\end{array}$ & $\begin{array}{l}\text { Faktor } \\
\mathrm{X}\end{array}$ & $\begin{array}{l}\text { Dade } \\
\text { Quick- } \\
\text { Wert }\end{array}$ & $\begin{array}{l}\text { Faktor } \\
\text { X }\end{array}$ & $\begin{array}{l}\text { Behring } \\
\text { Quick- } \\
\text { Wert }\end{array}$ & $\begin{array}{l}\text { Faktor } \\
\mathrm{X}\end{array}$ & $\begin{array}{l}\text { Thromt } \\
\text { Quick- } \\
\text { Wert }\end{array}$ & $\begin{array}{l}\text { borel } \\
\text { Faktor } \\
\text { X }\end{array}$ & $\begin{array}{l}\text { Simplas } \\
\text { Quick- } \\
\text { Wert }\end{array}$ & $\begin{array}{l}\text { stin } \\
\text { Faktor } \\
\text { X }\end{array}$ & $\begin{array}{l}\text { Boehrin } \\
\text { Quick- } \\
\text { Wert }\end{array}$ & $\begin{array}{l}\text { ger } \\
\text { Faktor } \\
\text { X }\end{array}$ & $\begin{array}{l}\text { Thromb } \\
\text { Quick- } \\
\text { Wert }\end{array}$ & $\begin{array}{l}\text { botest } \\
\text { Faktor } \\
\text { X }\end{array}$ & $\begin{array}{l}\text { RVV } \\
\text { Faktor } \\
\text { X }\end{array}$ \\
\hline $\begin{array}{l}1 \\
2 \\
3 \\
4 \\
5 \\
6 \\
7 \\
8\end{array}$ & $\begin{array}{l}21,3 \\
21,3 \\
20,5 \\
29,4 \\
35,4 \\
30,7 \\
31,4 \\
13,5\end{array}$ & $\begin{array}{l}13,3 \\
14,7 \\
14,0 \\
20,4 \\
39,0 \\
30,7 \\
21,1 \\
12,0\end{array}$ & $\begin{array}{l}23,3 \\
22,9 \\
22,1 \\
30,8 \\
38,5 \\
32,7 \\
32,3 \\
17,5\end{array}$ & $\begin{array}{l}16,3 \\
17,3 \\
15,3 \\
21,2 \\
36,3 \\
30,3 \\
25,9 \\
11,8\end{array}$ & $\begin{array}{l}17,1 \\
15,4 \\
12,2 \\
21,0 \\
26,3 \\
25,1 \\
23,4 \\
13,8\end{array}$ & $\begin{array}{r}11,4 \\
11,4 \\
10,2 \\
16,6 \\
29,0 \\
25,6 \\
18,1 \\
8,0\end{array}$ & $\begin{array}{l}17,9 \\
18,4 \\
16,2 \\
24,6 \\
27,8 \\
26,5 \\
27,8 \\
16,7\end{array}$ & $\begin{array}{r}11,4 \\
10,8 \\
11,1 \\
15,8 \\
27,0 \\
20,2 \\
16,9 \\
8,3\end{array}$ & $\begin{array}{l}22,9 \\
25,3 \\
24,5 \\
32,9 \\
39,7 \\
31,9 \\
34,5 \\
19,1\end{array}$ & $\begin{array}{l}14,4 \\
17,2 \\
15,4 \\
22,7 \\
48,8 \\
36,4 \\
25,0 \\
13,0\end{array}$ & $\begin{array}{l}26,9 \\
27,2 \\
26,0 \\
34,7 \\
42,0 \\
38,4 \\
35,2 \\
17,1\end{array}$ & $\begin{array}{l}15,9 \\
15,6 \\
14,3 \\
21,3 \\
33,9 \\
26,0 \\
22,9 \\
14,4\end{array}$ & $\begin{array}{r}11,3 \\
9,1 \\
9,0 \\
12,0 \\
18,3 \\
15,8 \\
17,1 \\
8,1\end{array}$ & $\begin{array}{r}7,9 \\
8,0 \\
7,8 \\
10,9 \\
16,0 \\
13,9 \\
17,5 \\
8,5\end{array}$ & $\begin{array}{r}11,6 \\
13,5 \\
10,0 \\
18,9 \\
27,8 \\
28,2 \\
19,2 \\
9,8\end{array}$ \\
\hline$\overline{\mathbf{X}}$ & 25,44 & 20,7 & 27,5 & 21,8 & 19,3 & 16,3 & 22,0 & 15,2 & 28,9 & 24,1 & 30,9 & 20,5 & 12,6 & 11,2 & 17,4 \\
\hline
\end{tabular}

tor $\mathrm{X}$ unterschiedliche Werte erhalten werden, wenn verschiedene Thromboplastine herangezogen werden. Bei diesèr Versuchsanordnung wäre es auch möglich, durch Verwendung von Russel's Viper Venom, das gegen nichtcarboxylierte Gerinnungsfaktoren besonders empfindlich sein soll $(10,11)$, einen thromboplastinunabhängigen Wert für die Wirkung nicht-carboxylierter Gerinnungsfaktoren zu erhalten.

Tabelle 4 gibt neben den Quick-Werten die entsprechenden Werte der Faktor X-Bestimmung der gleichen Plasmen (in Prozent der Norm), gemessen mit verschiedenen Thromboplastinen, an. Es ist zu ersehen, daß mit Throm: botest und den beiden Thromboplastinen der Behringwerke recht niedrige Faktor X-Aktivitäten bestimmt werden, die auch niedriger liegen als die Faktor X-Aktivitäten, dịe mit Russel ș Viper Venom ermittelt wurden. Die anderen Thromboplastine zeigen wesentlich höhere Werte an, wie Boehringer mit 20,5, La-Roche 20,7 und Dade mit 21,8. Ein bêsonders hoher Faktor X-Wert wurde mit Simplastin ermittelt. Auch hier wurden die auffälligen Unterschiede zwischen den verschiedenen Thromboplastinen im t-Test bestätigt (Daten nicht wiedergegeben). Bei den einzelnen Reagenzien liegen die Werte für die Faktor-X-Konzentration immer leicht unter dem Quick-
Wert des gleichen Plasmas. Eine deutliche Diskrepanz liegt nur bei dem Thromboplastin von Boehringer vor. Hier wird ein hoher Quick-Wert und eine niedrige Faktor $\mathrm{X}$-Konzentration gemessen.

Die Werte der Tabelle 5 zeigen nun, daß eine eindeutige Korrelation zwischen der Höhe des Quick-Wertes, dem Resultat der Faktor X-Bestimmung und dem errechneten Wert für nicht-carboxylierten Faktor X, gemessen

Tab. 5. Vergleich von Aktivität nicht-carboxylierter Gerinnungsfaktoren, Faktor X-Empfindlichkeit und Quick-Wert mit verschiedenen Reagenzien.

Die Werte sind den Tabellen 2 und 4 entnommen.

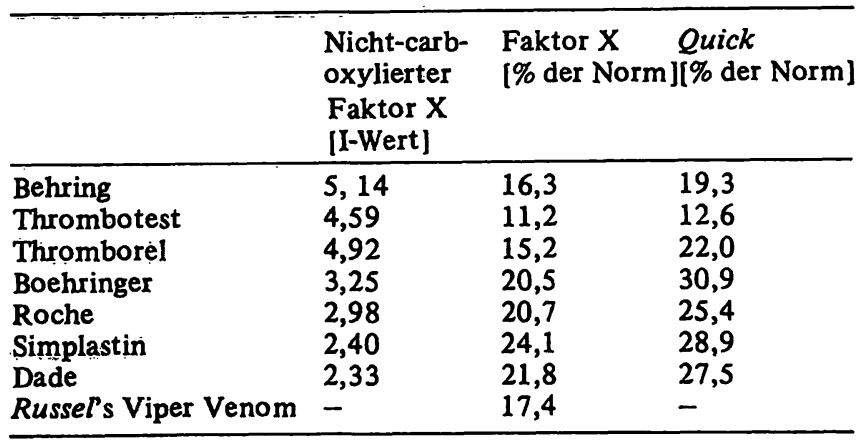


mit Reagenzien verschiedener Hersteller, existiert; das heißt, je empfindlicher ein Reagenz auf nicht-carboxylierten Faktor $\mathrm{X}$ reagiert, desto niedriger ist der mit diesem Reagenz gemessene Quick-Wert.

\section{Diskussion}

Im Plasma von Patienten, die mit Vitamin K-Antagonisten behandelt werden, liegen Gerinnungsfaktoren vor, die nicht oder nur unvollständig carboxyliert sind. Diese unvollständigen Faktoren besitzen eine veränderte Funktion. Sie beeinflussen das Testsystem, das allgemein zur Therapiekontrolle der Antikoagulation durch Vitamin KAntagonisten herangezogen wird, die Thromboplastinzeit. Über die quantitativen Veränderungen der Testsysteme durch die unvollständigen Faktoren ist wenig bekannt, ebenso wie über die Rolle, die sie bei der reduzierten $\mathrm{Ge}$ rinnungsfähigkeit des Blutes nach Applikation von Vitamin K-Antagonisten spielen.

Die Vitamin K-Abhängigkeit von vier Gerinnungsfaktoren ist bekannt: II, VII, IX und X.

Der Faktor IX wird durch die Thromboplastinzeit nicht mit erfaßt. Ebensowenig kann die nicht-carboxylierte Form in diesem Test interferieren.

Auf veränderte Faktor VII-Konzentrationen reagieren die Thromboplastine verschiedener Hersteller sehr unterschiedlich (12). Die Empfindlichkeit reicht aber bei allen Thromboplastinreagenzien aus, um die mit dem Faktor VII zusammenhängende Komplikation einer frühen Blutung, die mit der „Cumarinnekrose" einhergeht, rechtzeitig zu erfassen (13). Der nicht-carboxylierte Faktor VII reagiert nur unwesentlich langsamer als der intakte Faktor VII (14). Er hat auf die Thromboplastinzeit keinen wesentlich hemmenden Einfluß. Da der Faktor VII bei der Antikoagulation mit Vitamin K-Antagonisten als Faktor des Extrinsic-Systems von untergeordneter Bedeutung ist (15) - mit Ausnahme der schon erwähnten „Cumarinnekrose" (13) - haben wir keine eigenen Versuche zur Definition der Aktivität von nichtcarboxyliertem Faktor VII im Plasma so behandelter Patienten durchgeführt.

Eine Aktivität von nicht-carboxyliertem Faktor II läßt sich mit den in dieser Arbeit verwendeten Methoden (Verdünnung mit Mangelplasmen) nicht eindeutig definieren. Offensichtlich ist die Konzentration von vollständig carboxyliertem Faktor II im Plasma der antikoagulierten Patienten noch genügend hoch, so daß der Faktor II die Thromboplastinreaktionsgeschwindigkeit nicht wesentlich beeinflußt. Diese Ergebnisse stimmen gut mit denen von Löhliger (14) überein, der auch eine nur sehr geringe Aktivität von nicht-carboxyliertem Faktor II bei Verwendung mehrerer Thromboplastine nachweisen konnte, indem er die Differenz bei einer Faktor II-Bestimmung nach Verdünnung des Plasmas im Verhältnis $1: 10$ und $1: 20$ bildete.
Die einzige Möglichkeit, nicht-carboxylierten Faktor II isoliert zu erfassen, ist die Bestimmung mit Ecarin. Dieses Schlangengift ist in der Lage, auch das nicht-carboxylierte Prothrombin zu Thrombin zu spalten (16-18). Mit der Kombination Thromboplastinzeit/Ecarinbestimmung läßt sich die relative Konzentration von Prothrombin und nicht-carboxyliertem Faktor II ermitteln (18). Uber die klinische Relevanz einer solchen Bestimmung von nichtcärboxyliertem Faktor II herrscht noch Unklarheit (18).

Zur Beurteilung der Antikoagulation mit Vitamin K-Antagonisten werden Reagenzien bevorzugt, die Faktor Xempfindlich sind. Es ist nach den hier gezeigten Ergebnissen wahrscheinlich, daß mit den meisten Reagenzien nicht-carboxylierter Faktor $\mathbf{X}$ mit erfaßt wird. Auch ist es denkbar, daß die in vitro nachweisbare Hemmwirkung von nicht-carboxyliertem Faktor $\mathbf{X}$ in vivo bei der Herabsetzung der Gerinnungsfähigkeit des Blutes wesentlich ist. Wir finden im Plasma mit niedrigen Konzentrationen von Faktor $\mathrm{X}$ immer hohe Werte für nicht-carboxylierten Faktor $\mathbf{X}$ und mit Faktor $\mathbf{X}$ empfindlichen Thromboplastinen einen niedrigen Quick-Wert (Tab. 5). Dies bedeutet, daß die Konzentration von nicht-carboxyliertem Faktor X sich im Laufe der Antikoagulationsbehandlung ändert. Wir sind nicht der Meinung von Löhliger (14), der diskutiert, daß die Konzentration von nichtcarboxylierten Gerinnungsfaktoren unabhängig von der Antikoagulation ist. Nach unseren Beobachtungen sind die Werte für nicht-carboxylierte Gerinnungsfaktoren vom Ausmaß der Antikoagulation abhängig. Dies wird auch durch die Untersuchungen von Bertina (18) bestätigt, der für nicht-carboxylierten Faktor II eine unterschiedliche Konzentration dieses Proteins bei Patienten mit hohen oder niedrigen Faktor II-Werten gefunden hat; das heißt, bei niedriger Faktor II-Konzentration findet sich ein hoher Anteil an nicht-carboxyliertem Faktor II im Plasma.

In den vorliegenden Untersuchungen finden sich drei Reagenzien mit sehr hoher Empfindlichkeit gegen nichtcarboxylierten Faktor X: die beiden Thromboplastine der Behringwerke und Thrombotest.

Es ist möglich, daß die Thromboplastine der Behringwerke und auch von Thrombotest sowohl empfindlich gegenüber nicht-carboxyliertem Faktor II, als auch nichtcarboxyliertem Faktor X sind, weil mit diesen Reagenzien nach Verdünnung mit Puffer (Tab. 1) und Faktor X-Mangelplasmen hohe Werte gefunden werden. Es wird dabei angenommen, daß die Verdünnung mit Puffer (Tab. 1, Abb. 2a-2c) als Maß für die gesamte Aktivität nicht-carboxylierter Gerinnungsfaktoren gelten kann. Auch die gute Korrelation zwischen Faktor II-Konzentration, gemessen mit Thrombotest und nicht-carboxyliertem Faktor II (18), spricht für eine Empfindlichkeit von Thrombotest gegen nicht-carboxylierten Faktor II (18).

Es wurde lange Zeit angenommen, daß die kompetitive Hemmung durch nicht-carboxylierte Gerinnungsfạktoren nur mit dem Thrombotest-Reagenz erfaßt würde (1). In anderen Arbeiten wurde später dann auch anderen Rea- 
genzien eine Empfindlichkeit gegen dieses Protein zugestanden (19). In neueren Arbeiten (14) wird die Empfindlichkeit verschiedener Reagenzien gegenüber nicht-carboxyliertem Faktor $\mathbf{X}$ durch die Faktor $\mathbf{X}$-Bestimmung nach unterschiedlichen Verdünnungen $(1: 10 ; 1: 20$, s. u.) gemessen. Die erhaltenen Werte liegen zwischen 0,5 $\%$ (Thromboplastin aus menschlichem Hirn) und $11 \%$ (Simplastin). Ein Thromboplastin, das BCT (aus menschlichem Hirn), zeigt in dieser Versuchsanordnung (14) keine Hemmwirkung durch nicht-carboxylierten Faktor $\mathrm{X}$ an. In den hier vorliegenden Untersuchungen fanden wir eine Beeinflussung sämtlicher Reagenzien durch nicht-carboxylierten Faktor X, aber eine sehr große Variabilität der Empfindlichkeiten mit signifikanten Unterschieden (Tab. 3). Das BCT-Reagenz wurde nicht untersucht.

Unsere Werte stimmen mit den Ergebnissen anderer Arbeitsgruppen im Prinzip überein. So fanden wir bei Thrombotest einen durchschnittlichen I-Wert $(\bar{x}$ in Tab. 1) von 0,5 , bei Simplastin einen solchen von 0,4 . Eine ähnliche Relation wurde mit 1,0 bei Thrombotest und 0,7 bei Simplastin beschrieben (19). Ein direkter

\section{Literatur}

1. Hemker, H. C., Veltkamp, J. J. \& Loeliger, E. A. (1968), Thromb. Diath. Haemorrh. 19, 346-363.

2. Prydz, H. (1977), Seminars Thrombosis, Hemostasis 4, 1-11.

3. Morrissey, J., Jones, J. P. \& Olson, R. E. (1973), Biochem. Biophys. Res. Comm. 54, 1075-1082.

4. Nelsestuen, G. L. \& Suttie, J. W. (1972), Biochemistry 11, 4960-4964.

5. Shah, D. V., Suttie, J. W. \& Grant, G. A. (1973), Arch. Biochem. Biophys. 159, 483-491.

6. Stenflo, J. \& Ganrot, P (1972), J. Biol. Chem. 247, 81608166.

7. Stenflo, J. (1972), J. Biol. Chem. 247, 8167-8175.

8. Howarth, D. J., Brozovic, M., Stirling, Y. \& Reed, M. (1974), Scand. J. Haemat. 12, 346-354.

9. Loeliger, E. A. (1979), Thrombos. Haemostas. 42, 1141 1152.

10. Gaudernack, G. \& Prydz, H. (1975); Thrombos. Diath. Haemorrh. 34, 455-464.

11. Girolami, A., Müller, A. D. \& Hemker, H. C. (1972), Haemostas. $1,23-30$.

12. Averdunk, R. \& Braun, J. S. (1972), Blut $X X I V$, 303-313.

13. Loeliger, E. A. (190X), Anticoagulant induced haemorrhages. In: Blood disorders due to drugs and other agents (Girwood, R. H. ed.) Excerpta Medica, Amsterdam.
Vergleich der Werte ist nicht möglich, da die Höhe des IWertes von den verwendeten Plasmen abhängt. Besonders deutlich wird die Hemmung durch nicht-carboxylierten Faktor X, wenn mit Faktor X-Mangelplasma verdünnt wird. Bei einem solchen System fanden wir bei Thrombotest einen I-Wert von 4,6, während Simplastin einen solchen von 2,4 anzeigt.

Die Ergebnisse zeigen deutlich, daß die Quick-Werte reagenzienabhängig auf nicht-carboxylierten Faktor $X$ reagieren (Tab. 5). Dieses Ergebnis sollte zur Folge haben, daß bei einer Standardisierung des Quick-Wertes $(21,22)$ die Thromboplastine so verändert werden sollten, daß sie alle gleich empfindlich gegenüber nicht-carboxylierten Gerinnungsfaktoren sind und daß bei einer Standardisierung mit Referenzplasmen $(23,24)$ Plasmen von $\mathrm{Pa}$ tienten verwendet werden, die unter Phenprocumonbehandlung stehen und so nicht-carboxylierte Gerinnungsfaktoren enthalten.

Auch für die Richtigkeitskontrollen sollten bei den nichtcarboxylierten Faktor X empfindlichen Reagenzien Plasmen verwendet werden, die nicht-carboxylierten Faktor X enthalten.
14. Loeliger, E. A. \& Van Halem-Visser, L. P. (1969), Thrombos. Haemostas. 42, 1115-1127.

15. Quick, A. J. (1970), Thrombos. Haemostas. 23, 585-592.

16. Franza, B. R., Aronson, D. K. \& Finlayson, J. S. (1975), J. Biol. Chem. 250, 7057-7062.

17. Kornalik, F. \& Blombäck, B. (1975), Thrombosis Res. 6, 53-64.

18. Bertina, R. M., Van der Marel-Van Nieuwkoop, W. \& Loeliger, E. A. (1979), Thrombos. Haemostas. 42, 1296-1305.

19. Girolami, A., Brunetti, A. \& Patrassi, G. (1974), Blut 28 , $351-360$.

20. Loeliger, E. A., Taconis, W. K., Veltkamp, J. J. \& Hemker, H. C. (1970), Thrombos. Diath. Haemorrh. 23, 369-378.

21. Averdunk, R. \& Borner, K. (1970), Z. Klin. Chem. Klin. Biochem. 8, 263-269.

22. Poller, C. (1969), Brit. J. Haematol. 17, 606-613.

23. Miale, J. B. \& Lafond, D. J. (1969), Amer. J. Clin. Pathol. $52,154-161$.

24. Miale, J. B. (1962), J. Am. Med. Ass. 180, 736-743.
Professor Dr. R. Averdunk Klin.-chem. Zentallabor Klinikum Westend Spandauer Damm 130 D-1000 Berlin 19 
